\title{
ORNITONIMIA ISTRIANA: I NOMI DI TIPO MAZORIN PER LA SPECIE ANAS PLATYRHYNCHOS - UN RELITTO MEDITERRANEO?
}

\section{Premessa}

Nel presente saggio si cerca di interpretare gli ornitonimi di tipo mazurin come relitti mediterranei. i nomi popolari presentati fanno parte di un ampio corpus che abbiamo raccolto durante l'ultimo decennio nella regione istro-quarnerina (isola di Veglia compresa) in quasi 200 paesi per tutti gli idiomi istriani (istroveneto, istrioto, istrorumeno, i dialetti sloveni e croati e la parlata montenegrina di Peroj).

0.1 Per non confondere il lettore tutte le forme sono riportate con la stessa grafia: quella slovena con qualche segno specifico come $\int, \hat{z}, \hat{s}, 3$ ecc. (questi segni speciali si usano comunemente nei lavori di questo tipo, perciò non richiedono una particolare spiegazione) ${ }^{1}$. L'accento invece viene segnato come si usa nelle rispettive parlate, tranne per le parole istrorumene dove si adotta il sistema croato (semplicemente perché esso corrisponde alla realtà fonetica dell'istroromeno). Le località dove abiamo segnato l'ornitonimo vengono riportate tra parentesi nella lingua ufficiale della zona d'appartenenza, cioè o in croato o in sloveno.

\section{Identificazione biologica}

Il germano reale (Anas platyrhynchos) è una delle specie d'anitre (Anatidae) più comuni in Istria. Gjurašin all'inizio del secolo scrive: "Najmnogobrojnije su u hrvatskoj fauni zastupane medju svim plivačicama patke. Od svih njih prvo mjesto ide i radi broja u kom dolazi u našim zemljama, i radi toga, što nam je dala vrlo koristnu domaću pticu, patku divlju, ili kako je zove narod na prosto patka ili raca (...) a mužjaka patak, racak ili racman." (GJURAŠIN 302)

I sessi si distinguono chiaramente: "Maschio: capo verde metallico, stretto collare bianco, petto bruno porporino, parti inferiori grigio pallido, coda bianca con penne 
centrali nere arricciate, becco giallastro. Femmina: macchiata di bruno, becco bruniccio (spesso arancio ai lati). Entrambi sessi hanno largo "specchio" porpora viola compreso tra due strisce bianche (molto evidenti in volo) e zampe arancio." (PETERSON 56) "Il $\sigma$ in livrea nuziale si distingue facilmente dalla $\%$, screziata di bruno. Zampe rosso arancio, specchio delle ali blu, racchiuso tra due bande bianche. $\sigma$ in eclisse somiglia alla 9. (...) Vola con rapidi colpi d'ala, accompagnati da un fruscio ritmico. (...) Nidifica a terra sotto ai cespugli vicino all' acqua..." (DRCHAL 38)

\section{Gli ornitonimi}

\section{1.}

Nelle parlate istrovenete e istriote dell'Istria ${ }^{2}$ abbiamo segnato: màzor (Krk), mazorìn (Barban, Bertoki, Bivje, Galižana, Krasica, Strunjan, Višnjan, Vodnjan), màzoro (Barban, Krnica, Motovun), mazurìn (Brtonigla, Fažana, Funtana, Kanfanar, Labinci, Lovreč, Motovun, Oprtalj, Pićan, Plomin, Šišan, Tar, Vižinada, Vodnjan, Vrsar, Žminj), màzurin (Medulin), mazurìno (Brtonigla), màzuro (Buje, Galižana, Gradinje, Krnica, Lovreč, Lucija, Pirelići, Pula, Tar), maźurìn (Šverki), màzorin (Škrinjari), màzoro (Savudrija).

Solo a Umago (Umag) gli italofoni distinguono i sessi: màzuro ơ vs. màzura q - la forma femminile è un derivato in $-a$ dalla forma maschile.

Giglioli, p. 295, riporta mázorin e màzoro per il Veneto, mazorin per il Friuli e mazzurin per l'Istria. Boerio, p. 406, riporta tre forme per la nostra specie: màzaro, màzoro e mazorìn - l'ultimo ornitonimo riporta anche Pirona, p. 584, per le parlate friulane.

\section{2}

Le forme di questo tipo nelle parlate slave in Istria sono prestiti romanzi. Abbiamo raccolto: mầnzurin (Pomer), mần zurin (Medulin), màzarin (Ližnjan), mazorìn (Škatari, Štrped), mazurân (Praćana), mazurìn (Krk, Krušvari), mazurîn (Bale, Barban, Draguzeti, Gradinje, Kanfanar, Marčana, Pačići, Petehi, Plomin, Vrsar, Žminj), mazurìn (Baderna, Bankovići, Beletićev Brijeg, Blaškovići, Breg, Brovinje, Čepić, Čiritež, Filipana, Gradina, Katuni, Krasica, Krmed, Krnica Luka, Lovreč, Mali Mlun, Mavri, Motovun, Premantura, Premci, Rakalj, Režanci, Skitača, Soldatići, Tinjan, Veli Golji, Veli Mlun, Veli Vareški, Vidaci, Vodnjan, Vrh, Šumber, Žbandaj), mazurînac (Borut), ${ }^{3}$ mažurîn (Pićan), maźurìn (Foršići, Lupoglav) - nelle parlate croate; mazorìn (Dekani, Dragonja, Črni Kal, Izola, Koper, Korte, Marezige, Piran, Plavje, Pobegi, Prade, 
Sočerga, Škofije, Šmarje), mazurìn (Gračǐ̌če, Kubed, Šmarje), mazurín (Malija) - nelle parlate slovene; mazurìn - nella parlata montenegrina di Peroj.

2.3 .

L'istrorumeno mazurînu potrebbe essere un prestito istroveneto o ciacavo.

\section{La proposta etimologica}

L'analisi formale ci fa pensare all' etimo MAIOR, -ORE, REW 5247 che pensiamo sia da scartare, sebbene il contenuto "più grande" è giustificabile ("Tra le anatre di superficie è una delle più grandi /lunghezza $58 \mathrm{~cm} / "$ ") - DRCHAL 38. Bisogna dire però che molte altre specie hanno una lunghezza inferiore solo per un paio di centimetri ${ }^{4}$; cosa difficilmente visibile e in volo e sulla superficie d'acqua - cioè nelle situazioni comuni quando l'uomo (nel nostro caso il denominatore) incontra le anatre.

L'analisi semantica invece permette l'etimo MAS, MARIS "maschio", DIDL 633, che, nonostante l'impossibilità di giustificare formalmente le conservazioni di $-s-/-z-\mathrm{e}$ sim. nelle nostre forme, ci pare più accettabile. Dal punto di vista semantico il suddetto etimo è quasi obbligatorio: il popolo considera il maschio della nostra specie maschio par execellence di tutte le anatre ${ }^{5}$; ed infatti tutti i nomi popolari per la specie Anas platyrhynchos sono al maschile, invece per le altre specie sono quasi regolarmente al femminile. ${ }^{6}$

\section{1.}

Sebbene l'analisi formale richiede una $-r$ - al posto della $-s-/-z$ - e sim., siamo più propensi ad attribuire le forme di tipo mazurin ai relitti mediterranei che accettare il primo etimo citato. Purtroppo in questo momento non disponiamo di materiale lessicologico che potrebbe rafforzare la nostra ipotesi. Una prova, anche se debole, la troviamo in alcune forme istriane e venete che hanno conservato l'accento sulla prima sillaba (p. es. mànzurin).

4 Anas acuta - $55 \mathrm{~cm}$; Anas sterpera - $50 \mathrm{~cm}$; Anas penelope - $45 \mathrm{~cm}$; Anas americana - $55 \mathrm{~cm}$; Anas clypeata - $50 \mathrm{~cm}$; Netta rufina - $55 \mathrm{~cm}$; Aythya ferina $-45 \mathrm{~cm}$; Aythya marila $-47 \mathrm{~cm}$.

5 Gli altri maschi, di tutte le altre specie d'anatra, nella mente popolare in Istria sono considerati femmine.

6 Veramente ciò potrebbe anche esser dovuto al genere femminile del nome comune della famiglia: patka (croato), raca (sloveno), ana(i)tra (italiano). 


\section{ABBREVIAZIONI DELLE OPERE CITATE:}

BOERIO: G. Boerio, Dizionario del dialetto veneziano, Benetke 1856

DIDL: E. Bianchi - R. Bianchi - O. Lelli, Dizionario illustrato della lingua latina, Le Monnier, Firence 1982

DRCHAL: W. Černy - K. Drchal, Impariamo a conoscere gli uccelli, Istituto Geografico Deagostini, Novara 1982

GIGLIOLI: E.H. Giglioli, Avifauna italica, Le Monnier, Firence 1886

GJURAŠIN: S. Gjurašin, Ptice, I-II, Naklada "Matice Hrvatske", Zagreb 1899, 1901

PETERSON: R. Peterson - G. Mountfort - P.A.D. Hollom, Guida degli Uccelli d'Europa, Franco Muzio and c. Editore, Padova 1983

PIRONA: G.A. Pirona - E. Carletti - G.B. Corgnali, Il nuovo Pirona - vocabolario friulano, Società filologica friulana, Videm 1983

REW; W. Meyer-Lübke, Romanisches etymologisches Wörterbuch, Heidelberg 1972

Povzetek

ISTRSKA ORNITONIMIJA: MAZORIN (ANAS PLATYRHYNCHOS) - MEDITERANSKI RELIKT?

Prispevek obravnava ljudska imena za divjo raco (Anas platyrhynchos) tipa mazorin $v$ vseh istrskih govorih. Avtor predlaga kot koren besede lat. MAS, MARIS "samec". Čeprav formalna analiza ne dopušča predlaganega etimona (s formalno analizo pridemo do korena MAIOR "večji"), avtor meni, da je bolje predpostaviti mediteransko poreklo istrskih ornitonimov za divjo raco, kot opustiti prvi predlagani koren. 